BULL. AUSTRAL. MATH. SOC.

$04 A 05,03 B 10$

VOL. $29(1984), 365-376$.

\title{
MONADIC REPRESENTABILITY OF CERTAIN BINARY RELATIONS
}

\section{I.L. HUMBerstone}

Call a relation $R \subseteq A^{2}$ ( $A$ some non-empty set) monadically representable when there exist $F, G \subseteq A$ such that $R=\{\langle x, y\rangle \mid x \in F \circ y \in G\}$ for some truth-functional connective $\circ$. This note finds a first-order condition on $R$ which is necessary and sufficient for $R$ to be monadically representable.

Some relations are, we may feel, more relational than others. More specifically, in the case of binary relations, the satisfaction of a dyadic predicate by a pair of objects may simply be truth-functionally determined by whether or not the objects concerned satisfy (respectively) a pair of monadically expressible conditions; for example, given two monadic predicates $F$ and $G$ we could introduce the dyadic predicate $R$ by the definition: $F x y$ if and only if $(F x \& G y)$. While the defined predicate in such a case expresses a perfectly definite binary relation, we may still find the notation misleadingly to suggest a degree of connexion between the terms of the relation which the definiens reminds us is simply not there: for any objects $x$ and $y$, whether or not $x$ bears $R$ to $y$ is completely determined by whether or not these objects satisfy conditions separately stateable for $x$ and $y, x^{\prime}$ s satisfaction of $i t s$ condition being independent of all facts about $y$, and vice versa. In order to

Received 9 December 1983.

Copyright Clearance Centre, Inc. Serial-fee code: 0004-9727/84 $\$ \mathrm{~A} 2.00+0.00$. 
explore this particular dimension of spurious relationality (without denying there may be others), we define a binary relation $R$ (on some set $A$ ) to be monadically representable when, for some binary truth-functional connective $\circ$ there are sets (subsets of $A$ ) $F$ and $G$ for which it holds that for all $x$ and $y$ (in $A$ ) Rxy if and only if $F x \circ G y$. To avoid cumbersome circumlocutions, use-mention confusions of the type exhibited in this paragraph (as in using the same symbol for a predicate and its extension in some tacitly understood structure) will persist in what follows. The main purpose of this note is to find a first order condition on binary relations which is necessary and sufficient for their monadic representability, a purpose we shall find it possible to realize without using more than elementary logic in the proofs.

It is not hard to find conditions all monadically representable relations satisfy. An example would be the (universal closure of the) following:

$$
(R x y \& R v y \text { \& Ruz }) \rightarrow(R x z \vee R v v) .
$$

That all such relations satisfy (1) follows from the fact that for any binary truth-functional connective $\circ$, the schema (2) is tautologous:

$$
((A \circ C) \&(B \circ C) \&(B \circ D)) \rightarrow((A \circ D) \vee(B \circ E)) .
$$

To see this, note that if there is some assignment, $V$, of truth-values to $A, B, C, D, E$ on which the antecedent of (2) is true while the consequent is false, this implies that $V(A \circ C)=T$ while $V(A \circ D)=F$, so that. $V(C) \neq V(D)$; it also implies $V(B \circ C)=T$ while $V(B \circ E)=F$, so that $V(C) \neq V(E)$. Since there are only two truth-values, and $V(C)$ is distinct from each of $V(D), V(E)$, this means that $V(D)=V(E)$, which is incompatible with having $V(B \circ D)=T$ while $V(B \circ E)=F$, as the falsity on $V$ of the conditional also requires. Below, when it is claimed that a schema holds for all truth-functional choices of ' 0 ', an explicit justification will not be given, since only reasoning of the type just gone through suffices in each case. Theorem 2 of this note reports a certain strengthening of (I) to be both necessary and sufficient for monadic representability of the relation $R$. The proof uses information furnished by a more discriminatory account of the different modes of monadic representation, this information being supplied by Theorem 1 . The discrimination alluded to is over the choice of o used in the 
representation of $R x y$ as $F x \circ G y$. Before proceeding to Theorem 1 , however, it is appropriate to make a few comments in clarification of one aspect of the above definition of monadic representability.

The point deserving of special attention is that even when the predicate $F x$ in the definition of monadic representability is thought of as abbreviating some longer expression, the expression (open formula) in question should contain only the variable $x$ free; similarly with $G y$ and the variable $y$. (This follows from the definition since otherwise these formulae could not be thought of as having sets of individuals as their extensions: only relative to assignment of the remaining free variables would a set be determined.) We could call this the requirement of separation of variables, since what is being demanded of $R$ is that $R x y$ be equivalent to some truth-functional compound of two formulae in which compound the variables $x$ and $y$ are both free, but with free occurrences of these variables occurring in the separate components. This means we do not admit, for example, the following as showing the relation $R$ defined by

$$
R x y \leftrightarrow(E x \& E y) \vee(O x \& O y)
$$

to be monadically representable. Suppose the subject matter is the natural numbers, with ' $E$ ' and ' $O$ ' having for their extensions respectively the sets of even (or zero) numbers and of odd numbers, so that $R$ is the relation of agreement in parity (or congruence mod 2 ). Then we can put ' $\sim E^{\prime}$ in place of ' $O$ ' and so transform the above equivalence into one in which the requirement of separation of variables is met:

$$
R x y \leftrightarrow(E x \leftrightarrow E y)
$$

where $F=G='{ }^{\prime}$ '. In general the condition that the variables be separated across ' $\circ$ ' is a reasonable one to impose since otherwise the only obstacle to monadic representability would be the restriction to a finitary language, as we could always read off the appropriate representation from the graph of the relation. To illustrate this in the case where only finitely many ordered pairs belong to $R$, consider $R$ as pictured in Figure 1 : 


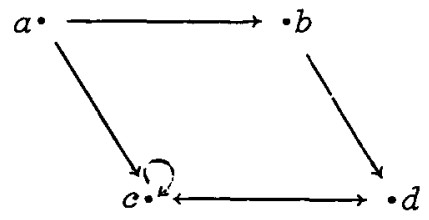

FIGURE 1

Choosing $A$ with extension $\{a\}, B$ with extension $\{b\}$, and so on, we simply set

$R x y \leftrightarrow(A x \& B y) \vee(A x \& C y) \vee(B x \& D y)$

$\vee(C x \& C y) \vee(C x \& D y) \vee(D x \& C y)$.

Returning now to the question of the choice of $\circ$, we introduce the following definitions. We say $R$ is \&-representable, v-representable, or $\leftrightarrow$-representable, according as for some $F$ and $G$ we have $\forall x, y(R x y \leftrightarrow(F x \circ G y))$ with 'o' respectively as ' $\&$ ', ' $v$ ' or ' $\leftrightarrow$ '. These special cases of monadic representability turn out collectively to exhaust the possibilities:

PROPOSITION. If $R$ is monadically representable it is either \&-representable, V-representable, or $\leftrightarrow$ representable.

This proposition is established by examination of cases, there being 16 binary truth-functions (in two-valued logic). It is simply a matter of checking that any representation in terms of one not figuring in the proposition can be rephrased in terms of one (or more) that does. For example, given

$$
\begin{aligned}
& R x y \leftrightarrow\left(F_{1} x+G_{1} y\right), \\
& R x y \leftrightarrow\left(F_{1} x \notin G_{1} y\right),
\end{aligned}
$$

or

$$
R x y \leftrightarrow\left(F_{1} x * G_{1} y\right),
$$

where $\phi$ and * have truth-tables:

\begin{tabular}{l|l|l}
$A$ & $\phi$ & $B$ \\
\hline$T$ & $T$ & $T$ \\
$T$ & $F$ & $F$ \\
$F$ & $T$ & $T$ \\
$F$ & $F$ & $F$
\end{tabular}

\begin{tabular}{l|l|l}
$A$ & $\star$ & $B$ \\
$T$ & $T$ & $T$ \\
$T$ & $T$ & $F$ \\
$F$ & $T$ & $T$ \\
$F$ & $T$ & $F$
\end{tabular}


(and $\rightarrow$ ' of course represents material implication), we put, respectively:

$$
\begin{aligned}
& R x y \leftrightarrow(F x \vee G y) \text {, where } G x \text { is } \sim F_{1} x \text { and } G y \text { is } G_{1} y, \\
& R x y \leftrightarrow(T x \& G y) \text {, where } F x \text { is }\left(F_{1} x \vee \sim F_{1} x\right) \text { and } G y \text { is } G_{1} y, \\
& R x y \leftrightarrow(F x \vee G y) \text {, with } F \text { and } G \text { as in the previous case. }
\end{aligned}
$$

In connexion with the three species of monadic representability isolated (though 'species' may be a misleading term since the classes are not mutually exclusive) it is here worth remarking that the converse of an $\&-, V_{-}$, or $\leftrightarrow$-representable relation is itself \&-, $\vee-$, or $\leftrightarrow-$ representable, respectively, while its complement is respectively $v-, \&-$, or $\leftrightarrow$-representable.

For the three modes of monadic representation distinguished here, we may define a corresponding trio of conditions on binary relations (outermost universal quantifiers having been omitted):

$$
\begin{aligned}
& {[\&]:(R x z \& R w y) \rightarrow R x y,} \\
& {[\vee]: R x y \rightarrow(R x z \vee R w y),} \\
& {[\leftrightarrow]:(R x y \leftrightarrow R x z) \leftrightarrow(R w y \leftrightarrow R w z) .}
\end{aligned}
$$

We make the sense of 'correspond' here explicit with

THEOREM 1. A relation $R$ is

(i) \&-representable if and only if $R$ satisfies [\&],

(ii) v-representable if and only if $R$ satisfies [v],

(iii) $\leftrightarrow$-representable if and only if $R$ satisfies $[\leftrightarrow]$.

Proof. (i) 'Only if'. Suppose $R x y \leftrightarrow(F x \& G y)$, for some choice of $F, G$. Then the claim follows from the fact that the expression

$$
((F x \& G z) \&(F w \& G y)) \rightarrow(F x \& G y)
$$

is a tautology.

'If'. Suppose $R$ satisfies [\&]. Define $F x \leftrightarrow \exists v(R x v)$ and $G y \leftrightarrow \exists v(R v y)$. Then $F x$ \& Gy implies the conjunction of the right-hand sides of these two equivalences, which in turn with the aid of $\exists-$ elimination and [\&], imply $R x y$. Conversely, that $R x y$ implies 
$F x$ \& Gy follows from the fact that two applications of $\exists$-introduction yield $\exists v(R x y) \& \exists v(R v y)$ from $R x y$.

(ii) 'Only if'. Suppose $R x y \leftrightarrow(F x \vee G y)$, for some choice of $F$, $G$. Then the claim follows from the tautologousness of

$$
(F x \vee G y) \rightarrow((F x \vee G z) \vee(F w \vee G y)) .
$$

'If'. Suppose $R$ satisfies [V]. Define $F x \leftrightarrow \forall v(R x v)$ and $G y \leftrightarrow \forall v(R v y)$. Then $F x \vee G y$ implies $\forall v(R x v) \vee \forall v(R v y)$, and so, instantiating ' $v$ ' to ' $x$ ' in the first disjunct and to ' $y$ ' in the second, Rxy . Conversely, Rxy implies $F x \vee G y$ in virtue of the condition [\&].

(iii) 'Only if'. Suppose $R x y \leftrightarrow(F x \leftrightarrow G y)$, for some choice of $F$, $G$. Then the claim follows from the fact that the following formula is a tautology:

$$
((F x \leftrightarrow G y) \leftrightarrow(F x \leftrightarrow G z)) \leftrightarrow((F w \leftrightarrow G y) \leftrightarrow(F w \leftrightarrow G z)) .
$$

(Note that each atomic formula appears exactly twice.)

'If'. We distinguish two cases.

(1) Suppose $\forall x \forall y(R x y)$. Then take $F$ and $G$ to be any coextensive predicates, and we have $R x y \leftrightarrow(F x \leftrightarrow G y)$.

(2) On the other hand suppose $\exists x \exists y(\sim R x y)$, and let $a$ and $b$ be such that $\sim R a b$. Now set $F x \leftrightarrow \sim R x b, G y \leftrightarrow R a y$. Then $F x \leftrightarrow G y$ implies $\sim R x b \leftrightarrow$ Ray and so $\sim(R x b \leftrightarrow$ Ray $)$. The condition $[\leftrightarrow]$ can be re-lettered, with re-grouping of subformulae, to

$$
(R x b \leftrightarrow R a y) \leftrightarrow(R a b \leftrightarrow R x y)
$$

The left hand side is false, so the right hand side must be, and since we have $\sim R a b$, it follows that Rxy . Conversely, Rxy implies $F x \leftrightarrow G y$, for suppose Rxy : then we have $R x y \leftrightarrow \sim R a b$, and $(R x y \leftrightarrow \sim R a b) \rightarrow(\sim R x b \leftrightarrow R a y)$ is a consequence of $[\leftrightarrow]$.

COROLLARY. From Theorem I, together with the proposition preceding it, we infer that a relation $R$ is monadically representable if and only if $i t$ satisfies the disjunction of the (universal closures of the) conditions [\&], [V], [њ].

Such disjunctions of universally quantified formulae as the 
characterization this corollary presents are somewhat hard to grasp, and when the universal quantifiers are 'prenexed' we find ourselves with a formula in twelve variables. In search of a more illuminating formulation, we return to the condition (1) which we noted earlier had to be met by any monadically representable relation. It may help to picture what this condition says thus :

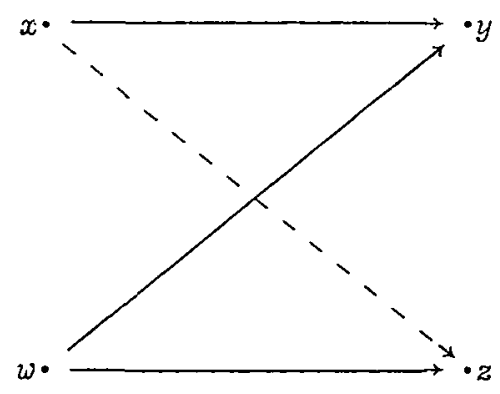

FIGURE 2

What condition (1) says is that if objects are related as by the solid arrows, then either the relation inducated by the dotted arrow obtains, or else the point $w$ in the diagram bears $R$ to absolutely everything. This suggests another condition on the relations we are interested in, involving not a universal $R$-bearer, but a universal 'R-target'. The condition will be that if a situation like that represented by Figure 2's solid arrows arises, then either the relation indicated by the dotted arrow obtains, or else everything bears $R$ to the point $y$; and indeed such a condition, here (3),

$$
(R x y \& \text { Ruy \& Ruz }) \rightarrow(R x z \vee \text { Ruy })
$$

must be met by any monadically representable relation $R$, in view of the fact that for any truth-functional choice of $\circ$, all instances of the following schema are tautologous:

$$
((A \circ B) \&(B \circ C) \&(B \circ D)) \rightarrow((A \circ D) \vee(F \circ B)) .
$$

Thus we can combine (1) and (3) into a single condition (backed by a single combined version - which need not be written out - of (2) and (4)):

$$
(R x y \& R v y \& R w z) \rightarrow(R x z \vee(R w v \& R u y)) .
$$

We have still not reached a sufficient condition for monadic 
representability, however. For (1) can be seen as one side of a coin of which (6), justified by the fact that no instance of (7) can fail to be tautologous, is the other:

$$
(R x z \& R w v) \rightarrow(R x y \vee R w y \vee R w z),
$$

$$
((A \circ D) \&(B \circ E)) \rightarrow((A \circ C) \vee(B \circ C) \vee(B \circ D)) ;
$$

and, parallelling (3) and (4), we have

$$
(R x z \& R u y) \rightarrow(R x y \vee R w y \vee R w z)
$$

backed by

$$
\{(A \circ D) \vee(E \circ B)\} \rightarrow((A \circ B) \vee(B \circ C) \vee(B \circ D)) .
$$

Finally, as with (1) and (3), we may combine (6) and (8) into

$$
(R x z \&(R v v \vee R u y)) \rightarrow(R x z \vee R v y \vee R v z) .
$$

We have, in the conjunction of (5) and (10), now reached a condition simpler than that afforded by the corollary to Theorem 1 which is both necessary and sufficient for monadic representability, this being the content of Theorem 2. In fact, noting the equivalence of each of (5), (10) to a formulation in which the variables ' $u$ ' and ' $v$ ' are identified, as well as the fact that a relation satisfies (10) if and only if its complement satisfies (5), the point we have reached is summarized somewhat more succintly in the statement of the theorem.

The proof of Theorem 2 will be aided by appeal to some pictures in the style of Figure 2, illustrating the typical situations involved in a failure of the conditions $[\&],[v],[\leftrightarrow]$. A failure of [\&] will involve objects related as in Figure 3:

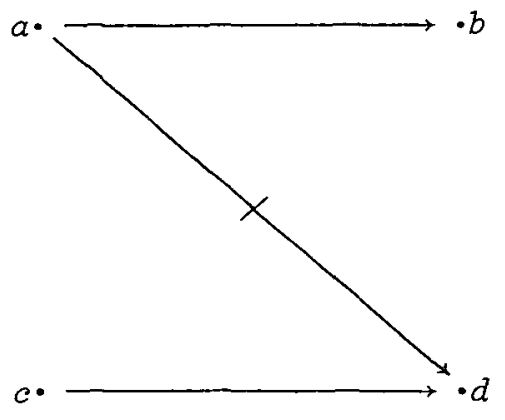

FIGURE 3 
On the other hand, for the condition [V] to fail, the following sort of situation must obtain somewhere in the structure:

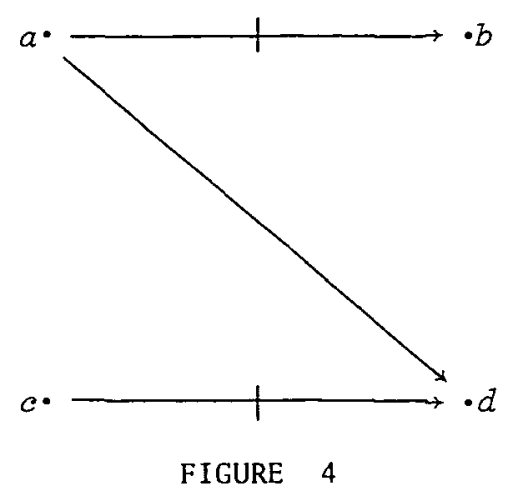

Finally, the condition $[\leftrightarrow]$ will be met unless either the sort of situation depicted in Figure 5a or else that depicted in 5b obtains (or both):

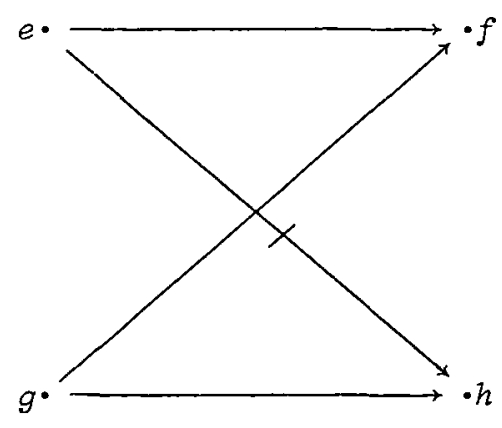

FIGURE $5 \mathrm{a}$

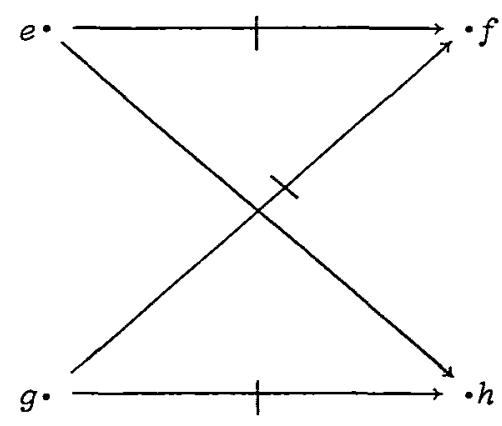

FIGURE 5b 
An arrow indicates that the relation $R$ holds between the object represented by the point at its tail and that represented by the point at its head (in that order), an arrow with a line through it indicating that the relation $R$ does not so hold. (So that, unlike the conventions in the theory of directed graphs, the absence of an arrow is simply non-commital with respect to the question of whether or not the relation holds.) The labelling of the points in these diagrams is purely for convenience of back-reference (in the course of the proof of Theorem 2), and it should perhaps be further emphasized that distinctness of points is not intended to signify distinctness of the objects they represent - except of course where that is entailed by the behaviour of $R$. Thus, for example, the condition $[\leftrightarrow]$ is also violated by the following configuration

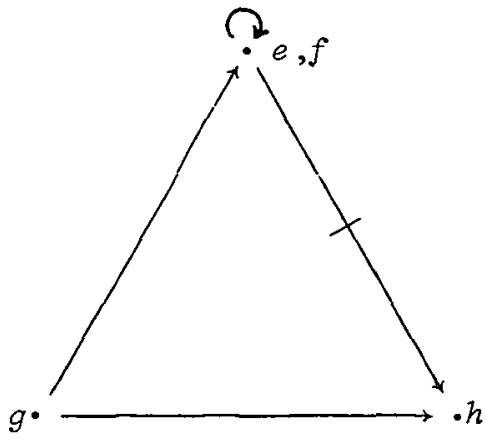

FIGURE 6

in which the points $e$ and $f$ (from Figure 5a) collapse to one. These explanations completed, we turn to

THEOREM 2. A relation is monadically representable if and only if it and $i$ ts complement satisfy the condition

$$
\forall w, x, y, z((R x y \& R v y \& R \omega z)) \rightarrow(R x z \vee(R v v \& R v y)) .
$$

Proof. 'Only if'. Already established in the course of discussing the conditions (5) and (10).

'If'. If $R$ is not monadically representable, then by the corollary to Theorem $1, R$ must exhibit violations of each of the three conditions $[8],[v],[\leftrightarrow]$. Focus on $[\leftrightarrow]$. Such a violation must take the form presented schematically in Figure $5 \mathrm{a}$ or else as in Figure 5b. We deal with the first case (5a) first. We note that such a violation of [↔] already 
involves a violation of [\&], so the remainder of the hypothesis that $R$ is not monadically representable is exhausted by suppose that [V] is also violated, as in Figure 4. The condition in the statement of the theorem implies via its consequence labelled (1) at the start of this note that the point $g$ in Figure 5 a bears the relation $R$ to everything, which we may put together with the information furnished by Figure 4 to infer that the situation is as pictured here:

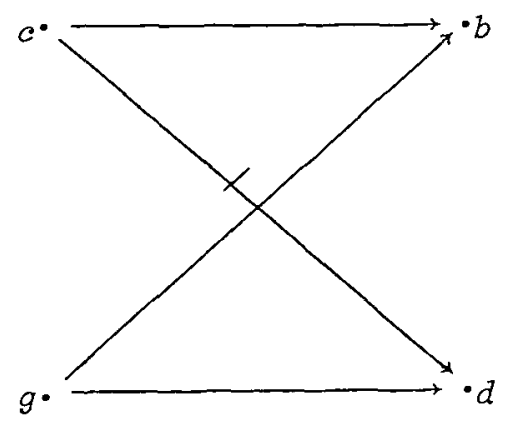

Therefore, by the condition of the theorem (more specifically, its consequence (3)) every element bears $R$ to $b$. This, however, contradicts another datum provided by Figure 4, namely, that $a$ does not bear $R$ to $b$. It remains to deal with the possibility that we have a violation of $[\leftrightarrow]$ of the type represented in Figure $5 \mathrm{~b}$. Here, a violation of $[v]$ is already evident and we make the additional supposition that [\&] is violated, as in Figure 3. This presents us with the complement of the relation $R$ holding exactly in those cases in which in the part of the proof just completed the relation $R$ held, so that since the condition in the theorem requires (5) to hold for the complement of $R$ as well as for $R$, a parallel argument goes through.

We conclude this note by observing that the phenomenon of reducibility of dyadic predicates to truth-functions of monadic predicates ramifies somewhat as we consider greater adicities. For example, one could investigate the monadic representability of ternary relations in the sense in which for ternary $S$ and monadic predicates $F, G$, and $H$, with $\circ$ some ternary truth-functional connective, the following counts as a madic representation

$$
\forall x, y, z(S x y z \leftrightarrow \circ(F x, G y, H z)),
$$

and one could also investigate the dyadic representability of such a 
relation, demanding only that there exist dyadic predicates $R_{1}, R_{2}, R_{3}$ for which we have

$$
\forall x, y, z\left(S x y z \leftrightarrow \circ\left(R_{1} x y, R_{2} y z, R_{3} x z\right)\right)
$$

However, neither generalization of the monadic representability of a binary relation (nor various 'hybrid' possibilities that also come to mind) will concern us here.

Department of Philosophy, Monash University,

Clayton, Victoria, 3168, Australia. 